\title{
LA INVESTIGACIÓN ACCIÓN PARTICIPATIVA PARA LA ORGANIZACIÓN COMUNITARIA EN GUADALUPE, MUNICIPIO JIMENEZ
}

\section{THE PARTICIPATORY ACTION RESEARCH FOR THE COMMUNITY ORGANIZATIONIN GUADALUPE, JIMENEZ MUNICIPALITY}

\section{RESUMEN}

Los grupos formados en la participación y comprometidos con la acción social, configuran un cuadro capaz de tomar decisiones que vislumbren soluciones a los problemas comunitarios. Debido a esto, se realizó una investigación cuyo propósito fue proponer la investigación acción participativa para la organización comunitaria en Guadalupe, Municipio Jiménez. La sustentación teórica se basó en la teoría crítica de Carr y Kemmis y aspectos de la investigación acción reseñados por Elliot. El estudio se enmarcó en el paradigma sociocrítico con la utilización del método de investigación acción participativa. La evidencia se recopiló a través de técnicas cualitativas: observación participante, entrevista no estructurada y grupo de discusión. La información fue analizada con el modelo Glater. Los hallazgos permitieron reconocer que la participación con la comunidad generó en los actores sociales la necesidad de organizarse, cambiar su realidad sobre la artesanía en madera, partiendo de sus recursos y del propio conocimiento popular. Conocimiento que mediante el proceso de investigación fue explicitándose, creciendo y estructurándose, por las acciones de los artesanos. Situaciones en las que el equipo investigador simplemente fue copartícipe en el desarrollo de una metodología que sirve de apoyo en la construcción de una estrategia de acción para el cambio.

Palabras clave: investigación acción participativa, participación, organización comunitaria.

\section{ABSTRACT}

The groups formed in participation and committed to social action, configure a cadre capable of making decisions that envision solutions to community problems. Due to this, an investigation was carried out whose purpose was to propose the participatory action research for the community organization in Guadalupe, Municipio Jiménez. The academic support was based on the critical theory of Carr and Kemmis and aspects of action research reviewed by Elliot. The study was framed in the sociocritical paradigm with the use of the Participatory Action Research method. The evidence was gathered through qualitative techniques: participant observation, unstructured interview, and discussion group. The information was analyzed with the Glater model. The findings allowed to recognize that the participants with the community generated in the social actors the need to organize themselves, to change their reality about the woodwork, starting from their resources and from popular knowledge. Knowledge that by means of the research process was being explained, increased and got structured by the actions of artisans. Situations in which the research team was simply a copartner in the development of a methodology that supports the construction of an action strategy for change.

Keywords: participatory action research, participation, community organization.
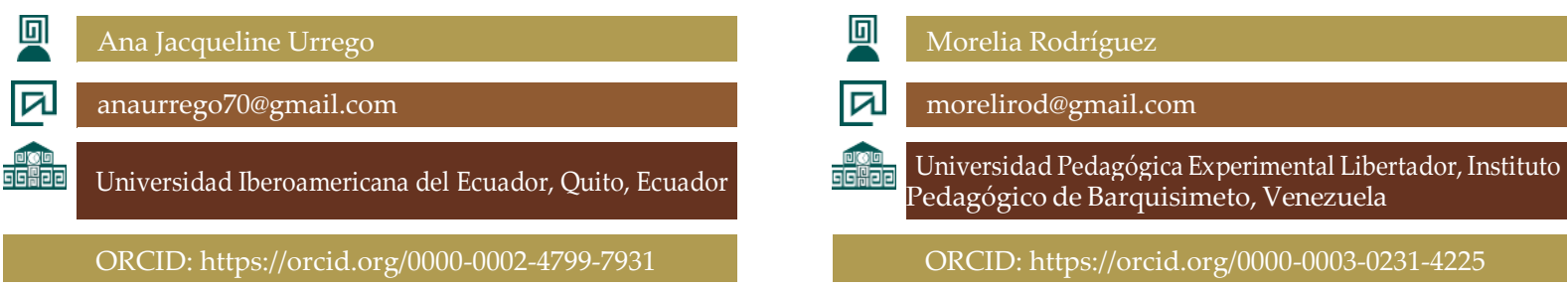


\section{INTRODUCCIÓN}

Las instituciones educativas forman parte de la comunidad y como tal, son también instrumentos que influyen en los cambios sociales, económicos, culturales $\mathrm{y}$ ambientales. Esto, mediante formas de acción que promueven la realización de actividades, organizando las estructuras existentes y fortaleciendo las vías comunicacionales entre comunidad e institución.

La unión de estos elementos genera mecanismos de realimentación social para el establecimiento de interrelaciones entre el papel que deben jugar las instituciones universitarias, en este caso específico, y las comunidades pertenecientes al contexto de dichas instituciones.

Partiendo de lo antes expuesto, las autoras del presente trabajo, docentes en la Universidad Pedagógica Experimental Libertador, Instituto Pedagógico de Barquisimeto eligieron este ámbito contextual del Estado Lara donde se encuentra la población de Guadalupe, Municipio Jiménez.

Para ello, cumpliendo con lo que Martí (2012) denomina etapa de preinvestigación, se identificaron algunos elementos relacionados con la comunidad de Guadalupe, entre ellos que dicha población tiene características muy definidas con proyección nacional del quehacer creativo artesanal desde hace muchos años, que como contexto comunitario posee, al igual que otras regiones del país, una identidad cultural que la nutre. Entre otros aspectos se encuentra la talla de madera, pues, además de ser una expresión artística, es una forma de trabajo para subsistir.

Los distintos tipos de talla los realizan dependiendo de la zona: los de Palo Negro, Pueblo Negro y Guadalupe Centro, tallan frutas; los de Quebrada Grande, tallan figuras humanas y de animales; y los de Buena Vista tallan flores y frutas. Sus talleres se localizan en sus propias viviendas y en puestos comerciales donados por la Alcaldía de Jiménez, ubicados a orilla de carretera. Allí se encuentran las manifestaciones artísticas que exhiben las destrezas y habilidades de los artesanos, pero sin la perspectiva de ver la pieza como un trabajo artístico, sino como un producto comercial.

Destaca en esta etapa de pre-investigación que los pobladores de Guadalupe se encuentran lejos de una búsqueda hacia un mercado artesanal que sirva para asegurar un bienestar de tipo social y económico, reflejado esto en la pobreza imperante en el aspecto físico de la mayoría de las viviendas. Resalta también la ausencia de una organización comunitaria como población artesanal y como comunidad en general, lo que representó la problemática a estudiar en la investigación realizada.

Dicha problemática se evidenció en las situaciones de carácter social, como: servicio de agua potable deficiente, dificultad para la adquisición de la materia prima (la madera), desertificación, ausencia de un plan de viviendas dignas, centros educativos con marcadas carencias, insuficiencia de módulos policiales, ambulatorios y otras.

En vista de lo anterior, se realizó un trabajo conjunto con grupos de artesanos del caserío de Guadalupe para la formación de los mismos como investigadores comunitarios, con miras al logro de acciones investigativas que permitieran la organización comunitaria. Para tal efecto, se planteó como objetivo: proponer la investigación acción participativa para la organización comunitaria en Guadalupe, Municipio Jiménez.

Para ello, se desarrollaron diferentes momentos de acción-reflexión-acción y se cumplieron con las características propias de la investigación acción participativa que establece Pring (citado por Latorre 2007), las cuales refieren aspectos cualitativos, recursivos, de participación y reflexión; porque se investiga tomando principalmente como fuente de información al lenguaje. Los pasos que se cumplen no son lineales, sino que se repiten cíclicamente según las necesidades, se involucra a los sujetos de la investigación, así como también se analizan y meditan situaciones en conjunto que llevan 
a nuevas acciones.

\section{METODOLOGÍA}

Las políticas de investigación de la Universidad Pedagógica Experimental Libertador, referidas al interés social de la investigación y la vinculación con el contexto universitario, sirvieron de base para la realización de este artículo de investigación producto de la experiencia vivida como coinvestigadoras en la población de Guadalupe, Municipio Jiménez.

Al respecto las políticas de investigación de la Universidad Pedagógica Experimental Libertador (2000:24) entre otros principios manifiesta que:

la función de investigación no estará guiada únicamente por el puro interés epistemológico, es decir por la búsqueda del saber como fin en sí mismo; o por la necesidad de apoyar y mejorar los programas docentes de pregrado, de postgrado y de extensión $\mathrm{o}$ por los intereses académicos o profesionales de la comunidad de estudiantes y profesores. Un criterio importante a considerar en la conformación de un programa de investigaciones, es elde interés social.

En lo antes expuesto, se refleja que la Universidad Pedagógica Experimental Libertador, genera acciones concretas para la interacción Universidad - Comunidad. Estas acciones se sustentan en tres premisas: la docencia como pilar fundamental en la formación, consolidación y rescate de los valores humanos. Asimismo, la investigación como apoyo al desarrollo técnico, humano y científico. Por último, la extensión como enlace en la relación institución y el contexto comunitario.

Lo anteriormente planteado se concreta a través de las políticas de extensión, en las que se promueven valores encaminados hacia el fortalecimiento del binomio: universidad- comunidad y específicamente en este estudio, tiene el propósito de facilitar el acompañamiento en la formación de estos investigadores comunitarios.

\section{PARADIGMA Y MÉTODO}

El proceso investigativo se realizósiguiendo el paradigma sociocrítico que según Murcia Florián (1992), se basa en la crítica social con carácter autorreflexivo; desde el cual el conocimiento se construye por intereses que parten de las necesidades de los grupos y sus problemas sociales e intentan transformar el contexto al iniciar un proceso investigativo que parte de situaciones consideradas problemáticas por los propios actores sociales.

En consonancia con el paradigma sociocrítico, el enfoque investigativo que se siguió fue el cualitativo, con la utilización del método de Investigación Acción Participante (IAP). Puesto que: "se pretende con la IAP destacar, por una parte, el producir conocimiento y acciones útiles para un grupo de personas; por otra parte, que la gente empodere/capacite a través del proceso de construcción y utilización de su propio conocimiento." (Rodríguez Gómez, Gil Flores \& García Jiménez 1996:56)

De la proposición anterior se puede justificar al método de la investigación acción participativa (IAP), al señalar que la labor del investigador local tiene un deber ser al propiciar "intercambios constructivos entre investigador y comunidad en los que se abordan conjuntamente todas las etapas del proceso investigativo y de intervención social." (Durston \& Miranda 2000:7).

De manera que el investigador local es aquel logra acceder al contexto de estudio con una intención de participación y no como un interventor externo puesto que la realidad contextual está construida simbólicamente por quienes en ella están inmersos. En el caso de Guadalupe, la figura de 
investigadoras locales sirvió para propiciar encuentros de discusión, debate y reflexión colectiva, encaminados en primer lugar al conocimiento que se fue generando producto de la interpretación de los hechos sociales. En segundo lugar, la recuperación paulatina del reconocimiento de cada participante como miembro de esa comunidad y, por último, la generación de procesos para transformar el entorno en un bien común.

\section{SUJETOS DEL ESTUDIO}

Para llevar a cabo el estudio se involucraron como sujetos de la investigación a 11 artesanos pertenecientes a la comunidad de Guadalupe, Municipio Jiménez, quienes manifestaron su deseo de participar. Según Rodríguez Gómez et al. (1996), la elección de informantes o sujetos del estudio se realiza de forma intencionada de acuerdo con los criterios establecidos por las condiciones que determine el investigador.

En este caso el criterio establecido fue el deseo voluntario de participar en el proceso de realización del estudio. El grupo estuvo conformado por: Héctor Rodríguez, Belkis de Medina, Corteza Rea, Esmeralda Guédez, José del Pilar Medina, Mónica Guédez, Pedro Mendoza, César Yépez, Moraima Rodríguez, Vicente Fréitez y Deibis Guédez.

\section{FASES DE LA INVESTIGACIÓN}

El proceso de investigación, se cumplió tomando en consideración las cuatro fases propuestas por Rodríguez Gómez et al. (1996). Estas fases fueron: Fase I. Preparatoria, Fase II. Trabajo de campo, Fase III. Fase analítica y Fase IV. Fase informativa. Las cuales se detallan a continuación:

Fase I (Preparatoria): se inicia esta fase, con la inquietud de las investigadoras por conocer a los pobladores de Guadalupe, y se concreta con las visitas realizadas al caserío. En este sentido, se comenzó con un recorrido a los diferentes sectores de la zona, para un reconocimiento de la misma y de sus habitantes, casi todos ellos artesanos.

Cabe destacar que este proceso de conocimiento del sector consistió en un diagnóstico, cuya etapa resultó de las conversaciones espontáneas entre los pobladores y la investigadora. De ahí que, la evidencia en esta fase se obtuvo mediante observaciones realizadas en distintas fechas, que sirvieron de información clave para el estudio.

Fase II (Trabajo de Campo): consistió en ir accediendo a la información básica para la investigación. En este sentido, se procedió a invitar a los habitantes de la comunidad de Guadalupe a una reunión a realizarse en la Casa de la Cultura, para conversar sobre diferentes tópicos de interés común y poder tener un acercamiento grupal con los mismos.

Dicha invitación se hizo extensiva por medio de algunos de los artesanos, quienes de boca en boca animaron a familiares, vecinos y amigos, para acudir y plantear los distintos problemas que aquejan a la comunidad en general. Luego de aceptada y confirmada la asistencia de los mismos, se procedió a realizar el encuentro con parte de la comunidad y días después, con artesanos más preocupados e involucrados en la problemática. Por lo que las investigadoras diseñaron el siguiente plan de acción en la tabla 1: 
Tabla 1: Plan de acción

\begin{tabular}{|c|c|c|}
\hline $\begin{array}{l}\text { Actividad } \\
\end{array}$ & $\begin{array}{l}\text { Objetivo } \\
\end{array}$ & Recursos \\
\hline $\begin{array}{l}\text { Visita a la comunidad de } \\
\text { Guadalupe. }\end{array}$ & Conocer la realidad geográfica. & $\begin{array}{l}\text { Humanos: Docentes } \\
\text { investigadoras. }\end{array}$ \\
\hline $\begin{array}{l}\text { Visita a la comunidad para } \\
\text { conversaciones espontáneas } \\
\text { con artesanos y pobladores. }\end{array}$ & $\begin{array}{l}\text { Diagnosticar la realidad a través de las } \\
\text { conversaciones con la comunidad }\end{array}$ & Guión de entrevista \\
\hline $\begin{array}{l}\text { Organización de encuentro con } \\
\text { la comumidad }\end{array}$ & $\begin{array}{l}\text { Organizar encuentro con la comunidad para } \\
\text { formación de equipo de investigación }\end{array}$ & $\begin{array}{l}\begin{array}{l}\text { Diario de campo, } \\
\text { lápices, } \\
\text { hojas. }\end{array} \\
\text { carpetas, }\end{array}$ \\
\hline $\begin{array}{l}\text { Consultar bibliografia sobre la } \\
\begin{array}{l}\text { Investigación } \\
\text { participativa } \\
\text { actividades }\end{array} \text { (IAP) en las } \\
\text { acción- }\end{array}$ & $\begin{array}{l}\text { Diseñar talleres sobre la IAP y su aplicación } \\
\text { como metodología de investigación en las } \\
\text { comunidades. }\end{array}$ & $\begin{array}{l}\text { Bibliografia } \\
\text { IAP. }\end{array}$ \\
\hline $\begin{array}{l}\text { Reuniones semanales con el } \\
\text { equipo de artesanos } \\
\text { investigadores }\end{array}$ & $\begin{array}{l}\text { Presentar la IAP como metodología de } \\
\text { investigación en la comunidad de artesanos. }\end{array}$ & Material fotocopiado. \\
\hline $\begin{array}{l}\text { Acompañar a los artesanos en } \\
\text { las actividades comunitarias } \\
\text { como visitas a entes } \\
\text { gubernamentales. a r enter }\end{array}$ & $\begin{array}{l}\text { Recopilar información a través de técnicas } \\
\text { etrnográficas. }\end{array}$ & $\begin{array}{l}\text { Grabaciones (audio) } \\
\text { Diario de campo. }\end{array}$ \\
\hline $\begin{array}{l}\text { Ejecutar taller sobre la IAP } \\
\text { como método de investigación. }\end{array}$ & $\begin{array}{l}\text { Presentar como método la IAP de } \\
\text { investigación }\end{array}$ & $\begin{array}{l}\text { Facilitadoras, } \\
\text { investigadoras } \\
\text { Artesanos }\end{array}$ \\
\hline $\begin{array}{l}\text { Organizar } \\
\text { recopilada. }\end{array}$ la información & $\begin{array}{l}\text { Categorizar la evidencia para identificar } \\
\text { cambios a partir de la IAP. }\end{array}$ & Modelo Glater \\
\hline $\begin{array}{l}\text { Reflexionar sobre las acciones } \\
\text { efectuadas con el equipo de } \\
\text { investigacion. }\end{array}$ & $\begin{array}{l}\text { Revelar las mejoras a partir de la IAP en la } \\
\text { transformación social. }\end{array}$ & $\begin{array}{l}\text { Humanos: Artesanosy } \\
\text { comunidad en general. }\end{array}$ \\
\hline
\end{tabular}

Fuente: Elaboración propia

Aunque en el plan de acción presentado en la tabla 1, se muestran de modo sucesivo una serie de acciones, no quiere decir que estas tienen un orden lineal en su ejecución, por el contrario algunas de esas acciones cumple un proceso cíclico, según la ejecución de la investigación.

Fase III (Analítica): se realizó sistematizando los momentos de la investigación, basadoen el modelo Glater, propuesto por González de Flores y Hernández Gil (2000), relacionados con los siguientes aspectos: (a) reducciónde evidencias y (b) organización de evidencias. Esto permitió a las investigadoras describir e interpretar las relaciones entre las diferentes manifestaciones comunicativas de los coinvestigadores en la ejecución del plan de acción.

Por consiguiente, en los encuentros sostenidos una vez cada quince días, durante meses con dichos artesanos, se aplicaron técnicas para tomar en cuenta las características individuales y su repercusión a nivel grupal en la búsqueda de soluciones a la problemática imperante en Guadalupe. Fase IV (Informativa): esta fase permitió la presentación y difusión de la información interpretada. De esta forma y en primera instancia se logró comunicar experiencias enriquecedoras a través de las vivencias compartidas, recalcando que se hicieron entregas a los coinvestigadores de la categorización de la información durante las fases, como un medio de confirmación de las interpretaciones enunciadas.

\section{TÉCNICAS E INSTRUMENTOS}

Asimismo, la recolección de la evidencia, se desarrolló con carácter flexible y espontáneo, entre coinvestigadores e investigadoras, las técnicas consistieron en las propias de la investigación cualitativa, como son: observación participante, testimonios focalizados y entrevistas en profundidad. Así como registros de notas en el diario de campo y la utilización de dispositivos de almacenamiento de audio y video.

\section{LEGITIMACIÓN DE LA INVESTIGACIÓN}

En cuanto a la legitimación de la investigación según Castillo (2003:52) se produce porque los datos "son realmente el resultado de una comprensión por parte del sujeto, colocando las interpretaciones en el contexto de la 'realidad vivida por éstos y en la óptica de una comprensión más válida del mundo social". En este sentido, la 
información recabada se legitimó a través de la triangulación de fuentes como técnica de confrontación que permitió interpretar la información de los sujetos de la investigación. Elliot (2000) acota que el principio básico que subyace a la idea de triangulación, es el de reunir observaciones e informes sobre una misma situación, efectuados desde diversas perspectivas, para luego señalarse los aspectos en los que difieren, coinciden $\mathrm{y}$ oponen.

En otras palabras, la triangulación permitió visualizar, al igual que la categorización, las distintas versiones planteadas por los sujetos. Dichas diferencias son las que dejaron entrever las posiciones asumidas por quienes estuvieron inmersos en la investigación, para comparar los puntos de vista de cada uno de ellos y así, llegar al análisis e interpretación de la información aportada por los actores sociales.

\section{ANÁLISIS DE LA INFORMACIÓN}

Una vez aplicadas las técnicas, el análisis de la información fue producido mediante el análisis e interpretación de las evidencias utilizando el Modelo Glater, creado por González de Flores y Hernández Gil (2000). Dicho modelo está conformado por los siguientes aspectos:

a. Código o temática, significa crear el código a las distintas temáticas para poder diferenciarlas.

b. Descripción, aquí se detallan las temáticas presentadas, pero tomando las propias palabras de los informantes.

c. Categoría, en este renglón el investigador agrupa la información obtenida dándole una denominación a cada grupo. Para distinguir las categorías emergentes de las preestablecidas se utiliza un símbolo diferenciador.

d. Interpretación, consiste en dar significado a la información recopilada, para ello se toma en cuenta la comprensión de las acciones humanas y se interpreta en función de las evidencias presentadas por los sujetos de la investigación.

La transcripción de los registros realizados con cada una de las técnicas: observación participante, entrevistas, testimonios focalizados y grupos de discusión, fueron los insumos para realizar el análisis a través de dicho modelo.

\section{RESULTADOS Y DISCUSIÓN}

En conversaciones promovidas con los pobladores de Guadalupe, se pudo identificar que la práctica artesanal se realiza junto algunos miembros de la familia, pero con la ausencia de una concientización del valor de su identidad como artesanos al no transmitir a las nuevas generaciones la importancia de preservar su memoria histórica, sólo transmitir la comercial. Asimismo, esta situación presente desde hace muchos años, se puede denominar como pérdida de valores en la memoria histórica colectiva, ya que, debido a la desorganización de la comunidad para resolver los problemas que la aquejan, el trabajo artesanal se convirtió en un trabajo individual, sin miras a un beneficio común hacia toda la población.

Lo anterior refleja la falta de políticas de los entes gubernamentales y de la propia población; es decir, existe conformación de una irregularidad social, económica y cultural que afecta en diversos sentidos la vida común y el quehacer de la comunidad de Guadalupe.

A continuación, a modo de ejemplo se presenta uno de los aspectos tratados en este modelo de análisis de la información: Desde esta perspectiva, la organización se percibió desde el ámbito comunitario como un sistema abierto al entorno, donde aprender es en primera instancia, desarrollar y superar situaciones que se dan en un contexto cambiante. Por consiguiente, una organización comunitaria efectiva requiere 
tratar con el contexto mediante la adaptación, el conocimiento sobre las relaciones interpersonales, y como elemento primordial la estructuración de acciones para propiciar soluciones. En la tabla 2, se presenta a modo referencia unos fragmentos del modelo Glater aplicado en la fase preparatoria:

Tabla 2: Acercamiento al escenario de estudio del contexto de Guadalupe

\begin{tabular}{|c|c|c|c|}
\hline $\begin{array}{l}\text { Códligo y } \\
\text { temática }\end{array}$ & Descripción & Categoría & Interpretación \\
\hline $\begin{array}{l}\text { OC } \\
\text { Organización } \\
\text { comunitaria }\end{array}$ & $\begin{array}{l}\text { El acercamiento para indagar } \\
\text { sobre los problemas que los } \\
\text { aquejan como comunidad, se } \\
\text { mició en la "casa de la Cultura" } \\
\text { de Guadalupe. En primera } \\
\text { instancia participaron algunos de } \\
\text { sus pobladores, la facilitadora } \\
\text { preguntó: } \\
\text { ¿Cuales son los problemas que } \\
\text { tienen aquí en Guadalupe? Al } \\
\text { respecto Héctor respondió. "Aquí } \\
\text { son muchos los problemas que } \\
\text { tenemos, pero para mi el } \\
\text { principal es la madera, porque de } \\
\text { eso vivimos" a lo que seguida- } \\
\text { mente José del Pilar acotó: "para } \\
\text { muchos de nosotros es que no } \\
\text { estamos organizados como } \\
\text { comunidad". } \\
\text { En ese momento toma la palabra } \\
\text { Corteza y dijo: "lo que pasa es } \\
\text { que nos interesa solo lo que } \\
\text { hacemos como artesanos, y no } \\
\text { nos importa lo que le pase al } \\
\text { vecimo". }\end{array}$ & $\begin{array}{l}\text { *Desorganización } \\
\text { comunitaria }\end{array}$ & $\begin{array}{l}\text { Las estrategias didácticas para } \\
\text { el desarrollo del acercamiento } \\
\text { con la comunidad, se } \\
\text { concentraron en el uso de la } \\
\text { técnica del grupo de discusión, } \\
\text { basada en la participación, } \\
\text { donde se generó un clima de } \\
\text { diálogo, para lograr una } \\
\text { manifestación espontánea sobre } \\
\text { la problematica que viven todos } \\
\text { como habitantes de Guadalupe. } \\
\text { Los participantes manifestaron } \\
\text { sus inquietudes, aunque unos } \\
\text { más participativos que otros, } \\
\text { pero en general interesados por } \\
\text { la temática. De alli, que dicha } \\
\text { manifestación pone en } \\
\text { evidencia que los mismos } \\
\text { resaltaran la importancia de la } \\
\text { IAP. Pues, esta permite } \\
\text { compartir saberes y al hacerlo } \\
\text { se nutren de sus propias } \\
\text { experiencias. De igual manera, } \\
\text { el intercambio de ideas se basó } \\
\text { en una reflexión critica, por lo } \\
\text { que se tomón en consideración } \\
\text { las inquietudes de cada uno de } \\
\text { los participantes. Se logró } \\
\text { construir el conocimiento, a } \\
\text { través del analisis y la } \\
\text { participación. }\end{array}$ \\
\hline
\end{tabular}

Fuente: Modelo Glater (González de Flores \& Hernández Gil 2000). Elaborado por las investigadoras a partir de la información recabada mediante el grupo de discusión. +Categoría preestablecida por las investigadoras. ${ }^{*}$ Categorías emergentes.

Como se aprecia en la tabla 2, se presentan algunas categorías y su interpretación en el denominado acercamiento al escenario de estudio. De hecho, en esos primeros encuentros los coinvestigadorescomenzaron a identificar la falta de organización comunitaria, como problema clave para la solución de los problemas que los aquejan, por lo que con la reunión de un grupo significativo de personas y con la atención prestada a cada uno de sus miembros, se buscó mediante su participación la solución a necesidades comunes. Situación que concuerda con la definición de participación comunitaria como "proceso social en virtud del cual, grupos específicos, que comparten alguna necesidad, problema o centro de interés y viven en una misma comunidad, tratan activamente de identificar esas necesidades, [...] adoptan decisiones y establecen mecanismos para atenderlas" (Aguilar Idáñez 2006:67).

Es de hacer resaltar, que a pesar del número de personas participantes (11 coinvestigadores), cuando se organiza el grupo comunitario se está organizando a la propia comunidad, pues la organización comunitaria como sistema, es un conjunto de partes independientes que interactúan entre sí, formando un todo y no un ente separado en sus partes (Mejía 2004). Este sentido de totalidad se conjuga como elementos vinculados de forma recíproca que influyen en las acciones de las personas, por ello, mientras más relacionados estuvieron como grupo, mayor fue su accionar.

Asimismo, uno de los aspectos más 
importantes de este estudio, fue tratar de entender la separación que puede existir entre la cultura propia de este sistema sociocultural cargada de símbolos con formas talladas en madera y la del resto del país, cuyos postulados deberían ser los de cuidar y querer lo autóctono.

Dentro de estos procesos colectivos, necesariamente hay que concebir a la organización como parte fundamental de la cultura y el modo más frecuente sería aprendiendo juntos, mediante el intercambio de conocimientos y experiencias que sirven para organizar a la comunidad a través de proyectos comunitarios, pero partiendo de un aprendizaje individual de toma de conciencia para el mejoramiento de todos los miembros, que efectivamente propició situaciones de cambio en su visión de organización.

Otras de las interpretaciones realizadas a la información recabada mediante las técnicas cualitativas se presentan en la tabla 3:
En los fragmentos de relatos presentados en la tabla 3, se dan indicios a la construcción del conocimiento fomentado mediante la IAP, corriente de la investigación acción en la cual todos los miembros participan en la mejora de la sociedad por medio de la investigación y se involucran en el aprendizaje permanente mediante una organización autogestora (Bisquerra 1996). En consonancia con la IAP, se realizó un proceso continuo de espiral en donde se analizaban los hechos y conceptualizaban los problemas, se planificaban y ejecutaban las acciones pertinentes y se pasaba a un nuevo proceso de conceptualización por medio de las reflexiones del equipo, por lo que la práctica colaborativa y la experiencia compartida constituyó la inserción de los participantes a tener una visión holística de su propia realidad.

Además, la actitud de los actores sociales de Guadalupe hacia la IAP, como método,

Tabla 3: Reflexión de las actividades realizadas

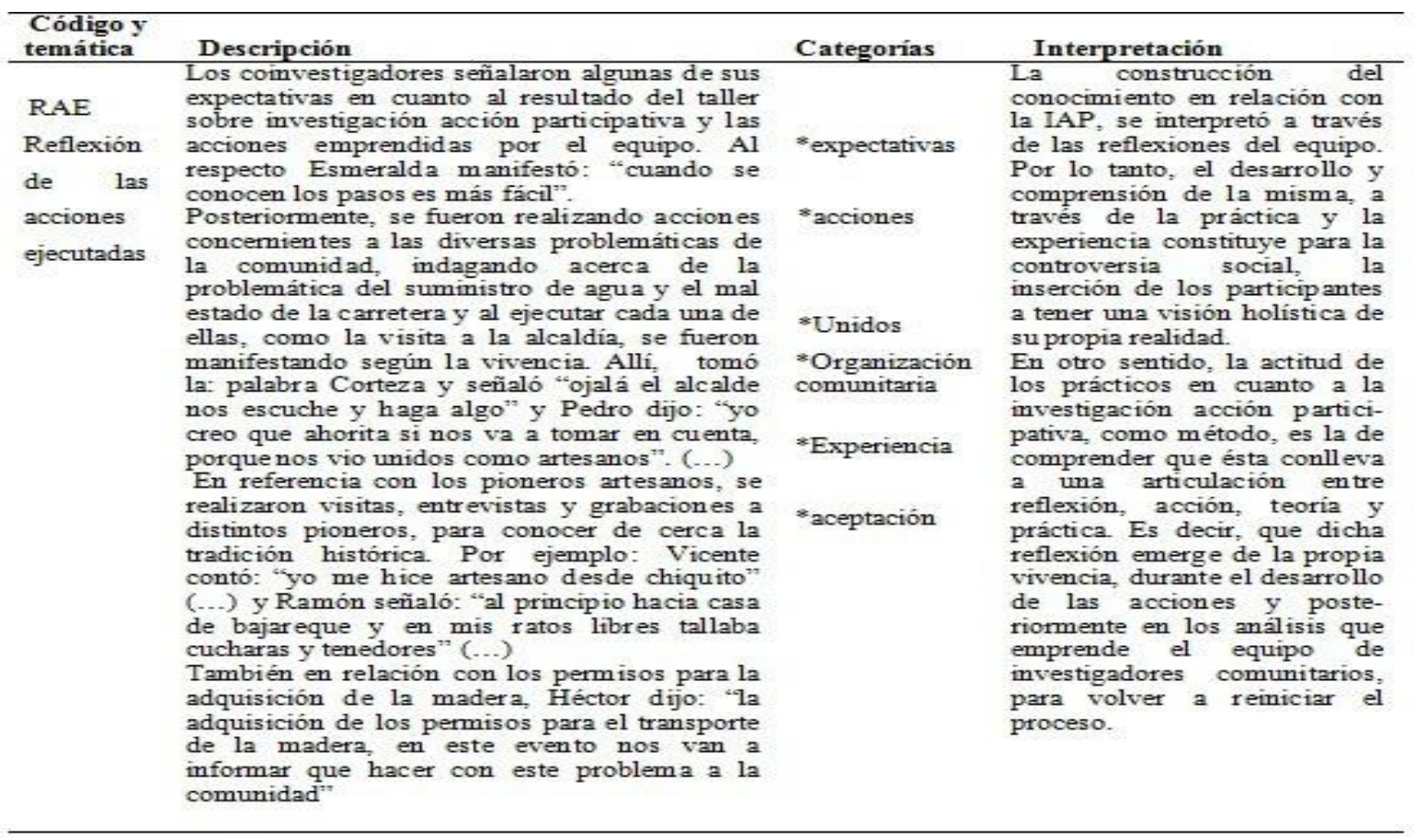

Fuente: Modelo Glater (González de Flores \& Hernández Gil 2000). Elaborado por las investigadoras a partir de la información recabada mediante entrevista y grupos de discusión. 
fue la de comprender que ésta conlleva a una articulación entre reflexión, acción, teoría y práctica. Destacando sus características producto de la construcción de una teoría, pero a la vez de una práctica que sirve para desarrollar una conciencia participativa. (Fals Borda \& Anisur Rahman 1991).

En otras palabras, en el entrenamiento formal de los investigadores sociales, por medio de la IAP, se consideró principalmente a la cultura de la gente común y las expresiones de la vida cotidiana. Además, se creó la posibilidad a través de un aprendizaje interactivo de ofrecer la oportunidad de reconstruir las debilidades que pudieran tener como individuos para así, reivindicar el derecho a interpretar su contexto social desde y por la comunidad, como sujeto protagónico de la misma. La interpretación emergió de la propia vivencia durante el desarrollo de las acciones y posteriormente en los análisis que emprendieron como equipo de investigadores comunitarios.

En efecto los actores sociales conformaron equipos con miras a la búsqueda de alternativas de solución a los problemas por ellos identificados como prioritarios. En tal sentido, el equipo del problema del agua indagó con algunas de las personas mayores de la comunidad cómo era en el pasado Guadalupe y el por qué no se había hecho nada al respecto, narraron algunas de las frases anotadas en sus diarios de campos (cuadernos), tales como lo expresado por Corteza: nos fuimos a preguntarle a María Chica sobre el tanque que estaba en la plaza hace muchos años y nos daba el agua. (C. Rea, comunicación personal, 17 de marzo, 2010)

Por otra parte, los integrantes del equipo de la adquisición de la madera para materia prima conversaron con el ingeniero, trabajador del Ministerio del Ambiente con sede en Quíbor, sobre la posibilidad de presentar un taller dónde se explicará a la comunidad la problemática existente con los permisos para las guías de movilización de la madera y las formas de proceder porparte de los artesanos. Este relato estuvo a cargo de Héctor expresando: El ingeniero nos atendió muy bien y creo que si hace el taller podemos informarnos de lo que tenemos que hacer para quitarnos ese problema de encima [...] y es mejor si trabajamos todos juntos en esto (H. Rodríguez, comunicación personal, 17 de marzo, 2010)

Esmeralda participante del grupo que indagó sobre el pasado histórico de Guadalupe y la autoestima del artesano, contó que realizaron una visita al Señor Cirilo Martínez, uno de los pioneros artesanos de la zona de Guadalupe hacia Quebrada Grande, allí lograron conversar de manera espontánea y el entrevistado expuso como comenzó su trabajo de artesano tallador. A esto ella refirió: a Cirilo lo conoce todo Guadalupe como pionero, es famoso hasta fuera de estos lares (E. Guédez, comunicación personal, 17 de marzo, 2010)

En este sentido, y a través de los relatos de las acciones emprendidas se visualizan las características de la investigación acción participante, las cuales se construyen de esta forma:

Primero, las problemáticas a estudiar surgieron de la propia comunidad. Segundo, el objetivo de la investigación es la transformación estructural y mejora de vida de la comunidad, como en efecto se inició el proceso en acciones antes descritas. Tercero, se implican personas en el lugar de trabajo controlando el proceso investigativo. Cuarto, se logra la sensibilización de las personas acerca de su compromiso como miembro de una comunidad $\mathrm{y}$, por último, el término investigador designa tanto a personas de la comunidad como los que cuentan con un entrenamiento especializado.

De ahí que esas actividades marcaron el inicio de una serie de acciones que por sus características no serían solucionadas en el transcurso de la investigación, pero si marcaron ciertos rasgos de organización para encontrar respuestas a sus planteamientos. Desde otro punto de vista, reconocieron que la autogestión resulta ser una herramienta básica para encaminar a la comunidad hacia la toma secuencial de la realidad compartida, 
que permite detectar e identificar situaciones que deben ser analizadas en sus partes, pero ubicándolas dentro de un todo que es el contexto del cual forman parte.

En tal sentido, la autogestión les permitió a las personas capacitarse en la medida que viven la experiencia de crecer como comunidad. Lo que desde la teoría crítica sería una posibilidad de emancipación, pues en efecto, "la teoría crítica constituye una guía para la acción humana, puesto que es una teoría emancipadora, que tiene contenido cognitivo, por tanto, es reflexiva. Es una teoría en la que prima el interés colectivo sobre el beneficio individual." (Naranjo Borja, Abad Merchán \& Ramos 2018:140). Por lo que el propósito de esta metodología investigativa sustentada en la teoría crítica fue brindar a los grupos con los cuales se trabajó, la posibilidad de lograr la autogestión y la participación para solucionar los problemas de la comunidad, pues "La participación hace del actor un promotor de acciones y generador de cambios, construyendo espacios que permiten actuar de distintas formas hasta lograr procesos de incidencia política y construcción social" (Castellanos Ramírez 2016:97). Todo ello a través del diálogo reflexivo y la toma de decisiones compartidas y comprometidas entre sus miembros.

De allí que, los artesanos participantes de la comunidad de Guadalupe, manifestaron llegar a sentir al contexto al cual pertenecen como un espacio de esperanza para impulsar la capacidad organizativa y la sustentabilidad. Esto a través de las alianzas logradas como equipo de investigación en relación con su comunidad y con los organismos competentes, para la búsqueda de mejoras a las que tienen derecho.

De igual manera, el artesano como protagonista de su propia realidad, se identificó a sí mismo como un ente con sentido de pertenencia hacia su comunidad. Además, capaz de reorientar a su comunidad dentro de una perspectiva consciente de los procesos de cambio necesarios para transformarla en el contexto situacional, social y cotidiano.

Es necesario resaltar que los hallazgos se ubicaron dentro de dos momentos: uno diagnóstico y otro referido a la ejecución del plan de acción.

El momento diagnóstico permitió reconocer y valorar la problemática del estudio, la cual estuvo referida a los problemas sociales que tienen como comunidad los artesanos de Guadalupe. Es decir, el habitante del caserío de Guadalupe reconocía las problemáticas que tienen como comunidad, pero de manera subjetiva, sin llevar a la práctica la organización de losinvolucrados. Esto ocurría por desconocimiento de los elementos de la IAP, hecho que se evidenció en los diferentes encuentros, cuando argumentó su aceptación de dichos eventos, pero sin entender el poder que tenían como grupo comunitario.

Además, según los propios participantes, la debilidad que tenían de enlazar la visualización de la problemática y de articularla con la práctica social, les impedía tomar decisiones como grupo, para cambiar la realidad social en la cual estaban inmersos. Por otra parte, el interés manifestado por todo el equipo de investigadores comunitarios, en conocer, indagar y aplicar la metodología de investigación permitió la elaboración de un plan de acción, acorde a las necesidades presentes en los distintos momentos de la misma. Dicho plan de acción les proporcionó las respuestas necesarias, durante todo el proceso de investigación.

En el momento de ejecución, la IAP, como metodología investigativa, permitió que el artesano del caserío de Guadalupe, reflexionara acerca de su realidad social, de sus problemas comunitarios y de la necesidad de formarse como investigador comunitario. Por tal motivo, se logró que el actor social dominara los aspectos teóricos y prácticos de la IAP (diagnóstico, plan de acción, ejecución y reflexión) y la relación que éstos tienen con su propia práctica social.

Además, el grupo logró entender la importancia de la IAP y de la comprensión epistemológica en los cambios que se 
pueden obtener a través del mismo y su interacción con la vida social comunitaria. De esta manera, "el esfuerzo por investigar, examinar, esclarecer la verdad ha implicado simultáneamente el trabajo de impugnar el error, la epistemología, como reflexión que busca la verdad, supone una reflexión sistemática" (Damiani 1997:31)

Así también, el apropiarse de la investigación acción les proporcionó a los actores sociales involucrados, las herramientas para aplicar las técnicas cualitativas en investigación, como son: entrevista en profundidad, testimonio focalizado, entre otras. De allí que, dentro de su formación como investigador comunitario, el artesano de Guadalupe reconoció que poseer diferentes tipos de fuentes de evidencias para validar la triangulación de la información, les facilitó la construcción de una práctica autogestionaria que iba en beneficio de sí mismo, pero principalmente en beneficio de toda la comunidad.

\section{CONCLUSIONES}

Las presentes reflexiones resumen un acercamiento a la realidad social estudiada, utilizando el método de IAP y técnicas cualitativas pertinentes, según el momento abordado por los investigadores comunitarios de la comunidad de Guadalupe. Dicho estudio se centró en los lineamientos instrumentados por la Universidad Pedagógica Experimental Libertador, a través del programa de postgrado y el deber ser de la actividad social, traducida en el acercamiento que libran hoy día las universidades hacia las comunidades. Las conclusiones de la experiencia investigativa se agrupan los siguientes ámbitos:

Ámbito Social: se propició y fomentó el trabajo en equipo, pues en este proceso investigativo se estimuló la colaboración entre los actores sociales para formarse como investigadores comunitarios. Estos encuentros sirvieron para exponer de manera espontánea sus inseguridades como artesanos, pero también el estudiar las posibilidades de lograr cambios dentro de su comunidad.

Se afianzó la reflexión en torno a situaciones desde una perspectiva socio critica, "basta presentar una explicación interpretativa, en la que se revele la posibilidad de definiciones y concepciones alternativas, para que los individuos, como si estuvieran esperándola, se vean inducidos a reinterpretar su situación y a modificar sus actos". (Carr \& Kemmis 1988:112). De esta manera, la IAP jugó un papel primordial en el crecimiento $\mathrm{y}$ desarrollo de la comunidad como grupo integrante de una sociedad, también un rol protagónico en los enfrentamientos dialécticos para la reconstrucción social, donde el ser humano puede expresarse de manera auténtica, siendo partícipe de su propia realidad.

Ámbito cognitivo: los actores sociales aprendieron a través de la técnica de la observación participante a observar y a ser observados, a tomar su cuaderno de notas para registrar con palabras propias sus reflexiones y por medio de los grupos de discusión, a expresarse como ente individual dentro de un grupo. Por lo tanto, las interpretaciones de su realidad se plantearon desde distintas perspectivas, pero siempre dentro de la investigación acción, es decir, los enfoques ontológico, epistémico y metodológico estuvieron presentes con un lenguaje cotidiano y sencillo, propios de su forma de ser.

Fue conveniente tener en cuenta también el contexto etnográfico, es decir las influencias de factores sociales, culturales e institucionales que tienen como grupo dentro de la comunidad. Por ende, redescubrir por medio de la IAP las herramientas y métodos legítimos, para hacer de la reflexión de su acción una fuente de información en la toma de decisiones para la transformación de su propia realidad.

Ámbito afectivo: se observó una disposición favorable hacia el desarrollo de los diferentes momentos de la investigación. Igualmente, 
hubo manifestaciones de crecimiento personal en cada uno de los participantes, lo cual propició una actitud crítica y autorreflexiva individual, que se reflejó en el comportamiento grupal.

Por otra parte, en lo afectivo, los coinvestigadores se conectaron con valores como: solidaridad, respeto y confianza, que implicó también mejorar como individuo y comprenderse como grupo, pues no se trata de una investigación hacia otras personas, sino sobre ellos mismos, en colaboración con otros implicados, ya que la finalidad última de la IAP es mejorar la práctica, al tiempo que se mejora la comprensión que de ella se tiene.

Resulta importante acotar que se logró transformar en parte la realidad social del artesano de Guadalupe, ya que se fusionó el quehacer educativo con la acción social. En otras palabras, los procesos reflexivos sirvieron de apoyo para la transformación social, mediante la integración de los procesos educativos con la cotidianidad de los artesanos. Esto permitió formar a los artesanos en investigadores sociales organizados para reflexionar sobre su práctica, haciendo uso de técnicas para la interacción dialógica y la sistematización de sus experiencias que le permitirán ir instrumentando acciones de mejora progresivamente.

\section{REFERENCIAS BIBLIOGRÁFICAS}

Aguilar Idáñez, M. J. (2006). Participación comunitaria en salud: balance crítico sobre los consejos de salud y otras experiencias participativas en Atención Primaria. Revista Comunidad, (9). Recuperado de http://www.pacap.net/ es/publicaciones/pdf/comunidad/9/ documentos-3.pdf

Bisquerra, R. (1996). Métodos de Investigación Educativa. Barcelona, España: Grupo Editorial Ceac.
Carr, W. \& Kemmis, S. (1988). Teoría Crítica de la enseñanza. Barcelona, España: Ediciones Martínez Roca S.A.

Castellanos Ramírez, L. (2016). Desarrollo rural: caso vereda Requilina en la localidad de Usme, Bogotá Colombia. Chakiñan, (1), 93-100. Recuperado de http://chakinan.unach.edu.ec/index. php/chakinan/article/view/14/11

Castillo, E. (2003). Lo científico de la investigación cualitativa: viejos dilemas, nuevas posturas. Nómadas,(18),46-53. Recuperado de https://www.researchgate.net/ publication/237022368_Lo_cientifico_ de_la_investigacion_cualitativa_ viejos_dilemas_nuevas_posturas

Damiani, L. (1977). Epistemología y Ciencia en la Modernidad. Caracas, Venezuela: Ediciones de la Biblioteca de la Universidad Central de Venezuela.

Durston, J. \& Miranda, F. (2000). Experiencias y metodología de la investigación participativa. Recuperado de https://repositorio.cepal.org/ bitstream/handle/11362/6023/6/ S023191_es.pdf

Elliot, J. (2000). La investigación acción en la escuela. Madrid, España: Morata.

Fals Borda, O. \& Anisur Rahman, M. (1991). Acción y conocimiento. Colombia: CINEP.

González de Flores, G. \& Hernández Gil, T. (2000). Análisis e Interpretación de la información en la Investigación Cualitativa. Barquisimeto, Venezuela: UPEL- IPB, Subdirección de Extensión Oficina de Promoción y Difusión.

Latorre, A. (2007). La investigación-acción. Conocer y cambiar la práctica educativa. Barcelona, España: Grao.

Martí, J. (2012). La investigación - acción participativa. Estructura y fases. Recuperado de: http:// www.redcimas.org/wordpress/wpcontent/uploads/2012/08/m_JMarti_ IAPFASES.pdf

Mejía, A. (2004). La Participación Ciudadana. Caracas, Venezuela: 
Biblioteca Básica Temática. Taller de diseño gráfico, ONAC.

Murcia Florián, J. (1992). Investigar para cambiar. Bogotá, Colombia: Cooperativa Editorial Magisterio.

Naranjo Borja, E., Abad Merchán, A. \& Ramos, V. (2018). Factores culturales de logro del sistema de producción comunitaria de la Parroquia Salinas en la Provincia de Bolívar, Ecuador. Chakiñan, (6), 136-148.Recuperado de http://chakinan.unach.edu.ec/index. php/chakinan/article/view/208/151

Rodríguez Gómez, G., Gil Flores, J. \& García Jiménez, E. (1996). Metodología de la Investigación Cualitativa. Málaga, España: Ediciones Aljibe.

Universidad Pedagógica Experimental Libertador. (2000). Políticas de investigación. Consejo Universitario. Caracas, Venezuela: FEDEUPEL. 